\title{
Active Cooling Camera Modification for Improved Astronomy Data Collection ${ }^{1}$
}

\author{
Chris Christopherson \\ Department of Physics and Astronomy \\ University of Wisconsin Oshkosk \\ Oshkosh, WI 54901
}

\begin{abstract}
Digital Single Lens Reflex (DLSR) cameras modified to be sensitive into the infrared are a relatively inexpensive entry into astrophotography. Such a camera was used for wide-field, narrowband observations of the Cygnus Superbubble in the Observing Nebulosities project (Christopherson and Kaltcheva, 2015). One of the downfalls to using a DLSR for such a study is the buildup of heat in the sensor during data collection and the noise introduced into the images as a result. For this project an active cooling modification was designed and installed on a DLR camera allowing its sensor to be cooled and held at a constant temperature below ambient conditions to reduce noise and speed up the data collection process.
\end{abstract}

\section{Introduction}

The Observing Nebulosities project (Christopherson and Kaltcheva, 2015) used a DSLR camera modified to capture wide-field near-infrared images using narrowband filters in Hydrogen- $\alpha$, Hydrogen- $\beta$ and Oxygen-III. We collected images of a large star-forming complex in the Milky Way, the Cygnus Superbubble, to obtain homogeneous coverage of the region. This allowed the study of the overall distribution of the interstellar gas as well as the ability to trace areas containing supernova shock fronts or strong stellar winds. Caplan and Deharveng (1985) and Cox, Deharveng and Caplan (1987) show that photometry in the Balmer emission lines, Hydrogen- $\alpha$ and Hydrogen$\beta$, can be used to study dust associated with star-forming regions. Using the work of Gruendl et al. (2000), observations using Hydrogen- $\alpha$ and Oxygen-III can be used to better understand the physical conditions of nebulae around very energetic stars. Collecting data for a project such as this requires many hours of exposure per filter to bring out the details in the object being studied. This is accomplished by taking many shorter exposures, five- to ten-minute for the Observing Nebulosities project, along with calibration images and summing the corrected results.

Collecting long exposure images for astronomy requires a set of calibration images to be taken along with the science images; they are: flat frames, dark flat frames, and dark frames. Flat frames are used to correct the science frames by removing vignetting and dust specs from the images. Dark flat frames remove sensor noise and stuck pixels from the flat frames. Dark frames are taken to remove heat noise and stuck pixels from the science frames. For the dark and dark flat frames to be effective, the calibration images must be taken with the same settings and under the same temperature conditions as the images they are correcting (Starizona). The most difficult aspect of collecting data for the Observing Nebulosities project was maintaining a constant imaging sensor

\footnotetext{
${ }^{1}$ Research done with the support of the Wisconsin Space Grant Consortium, NASA Space Grant College and Fellowship Program, NASA Training Grant \#NNX14AP22H
} 
temperature throughout a night of observations. Thus, many of the light frames from the first few nights of observing were unusable due to a lack of matching calibration frames. To keep the image sensor close to the same temperature throughout a night of observing a cool-down time equal to the exposure time was introduced between images (Christopherson and Kaltcheva, 2015). As the ambient temperature dropped through the night the temperature of the image sensor would drop as well. When the temperature dropped more than four degrees Celsius, more dark frames were taken to be able to correct the lower temperature science frames.

Although this method worked, the process was very inefficient and left me wanting to speed up the data collection process as well as keep the camera at a more constant temperature. Having seen commercial astronomy cameras with thermoelectric cooling and the ability to liquid cool their sensors led me to decide to design and build an active cooling modification for my DSLR camera. The plan was to make a modification that would be easy to install, maintain a constant sensor temperature below ambient conditions and minimize any pause between exposures.

\section{Design}

The active cooling modification consists of: a 3D-printed replacement of the rear housing of the camera (Fig. 1), heatsinks (Fig. 2), a computer CPU liquid cooling unit shown in (Fig. 3), a 60W thermoelectric (TEC) module, digital temperature and humidity sensors, $12 \mathrm{~V}$ relay module and an Arduino Mega 2560 microcontroller. The system was designed to be a direct replacement for the rear housing of the camera allowing for a simple installation of the upgrade.

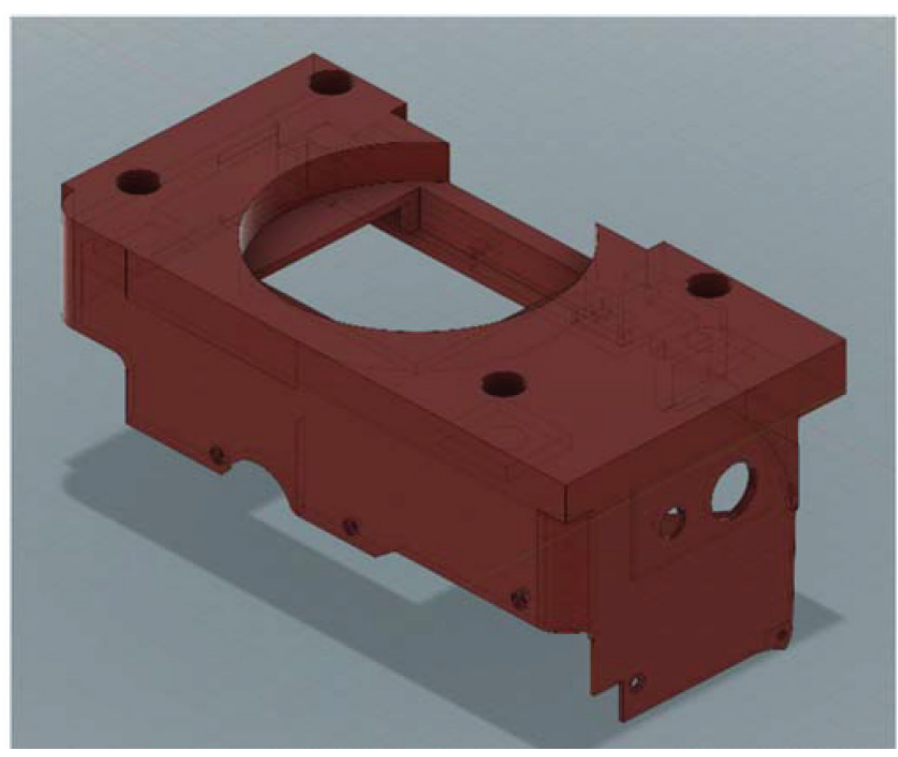

Fig. 1:Model of the 3D printed replacement rear housing of the DSLR camera

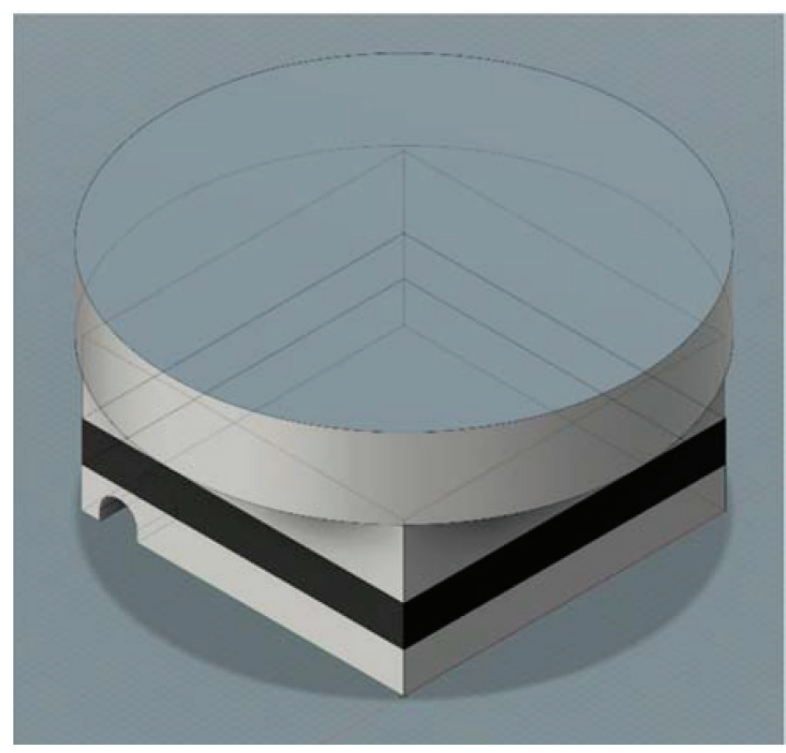

Fig. 2: Model of the heatsinks (gray) and thermoelectric cooler (black) placed between the camera sensor and water-block (not shown) 
The replacement rear housing shown in Figure 1 was designed to be attached to the camera using the same screw holes that held the original rear housing. Two side holes were added to connect the TEC to power and a digital DS18B20 temperature sensor. The top of the model in Figure 1 has a hole to allow a heatsink to pass through as well as four bolt holes to mount the liquid cooling system's block (Fig. 3) to the heatsink. The heatsinks (Fig. 2) were machined out of two pieces of aluminum. The top piece connects the water block of the liquid cooling system

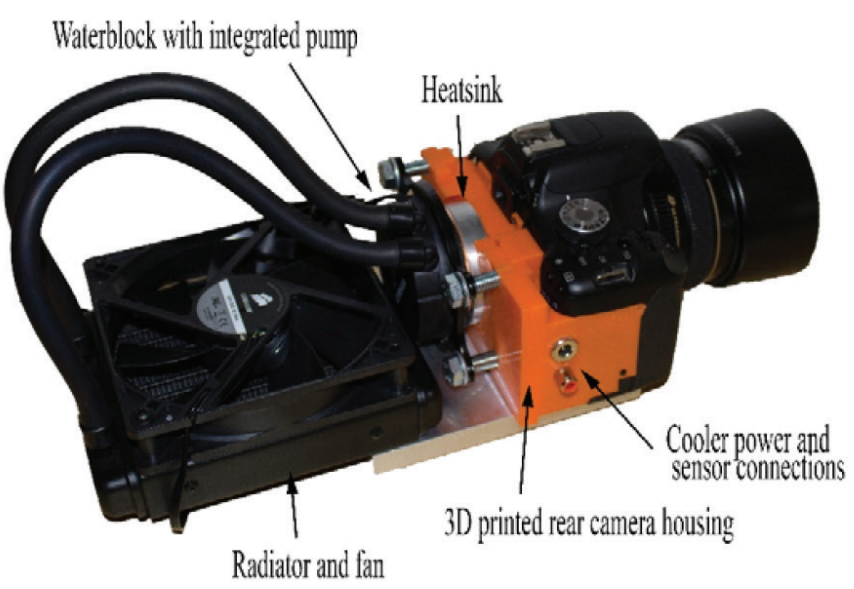

Fig. 3: DSLR camera with cooler modification installed to the TEC while the bottom connects the TEC to the back of the image sensor and houses a DS18B20 temperature sensor.

The Arduino Mega controls the electronics of the project and three external Arduino libraries (Arduino HDC1000 (Fried 2016), One Wire (Studt, 2014) and Dallas Temperature Control (Burton, 2016)), were used to allow the control of the temperature and humidity sensors. Temperature and humidity sensors are used to monitor ambient temperature and humidity, as well as set the desired image sensor temperature at startup. The Arduino is programmed to enable a $12 \mathrm{~V}$ relay to power the TEC module to cool the image sensor to the desired temperature by monitoring a DS18B20 temperature sensor placed in the heatsink described above. A cooling fan attached to the water cooler radiator and the water cooler pump are currently connected directly to the $12 \mathrm{~V}$ power source but will eventually be controlled by a Pulse Width Modulated (PWM) signal to allow the control of the fan and pump speed.

The cooling system, shown installed in Figure 3, removes heat from the camera's image sensor using the TEC module. The heat from the TEC module is then transferred through the heatsink to the block of the liquid cooling system, which pumps the liquid from the water block to the radiator where it is cooled with the assistance of the fan.

\section{Results}

Testing of the cooler resulted in a significant drop in sensor temperature, thermal noise in the test exposures and the removal of the cooldown time between images adopted during the Observing Nebulosities project. Figure 4 shows the temperature evolution of the unmodified camera sensor, taking five-minute Dark Frames with no pause between images, over the course of two hundred and fifty minutes. During the test, the image sensor temperature rose from $21 \mathrm{C}$ to $36 \mathrm{C}$ with an ambient temperature of 20C. The test of the cooling system, shown in Figure 5, was conducted by cooling the sensor to an initial temperature -10C and taking five-minute Dark Frames for two hundred and fifty minutes with no pause between exposures. While taking the Dark Frames for this test, the ambient temperature was recorded at $23 \mathrm{C}$ and after the first frame the senso $\mathrm{r}$ temperature read between $-8 \mathrm{C}$ and $-9 \mathrm{C}$, more than thirty degrees Celsius below ambient temperatures. Taking 
the difference in ambient temperatures between tests into account, the cooler maintained the image sensor temperatures at $46, \mathrm{C}$ below the maximum uncooled camera temperature.

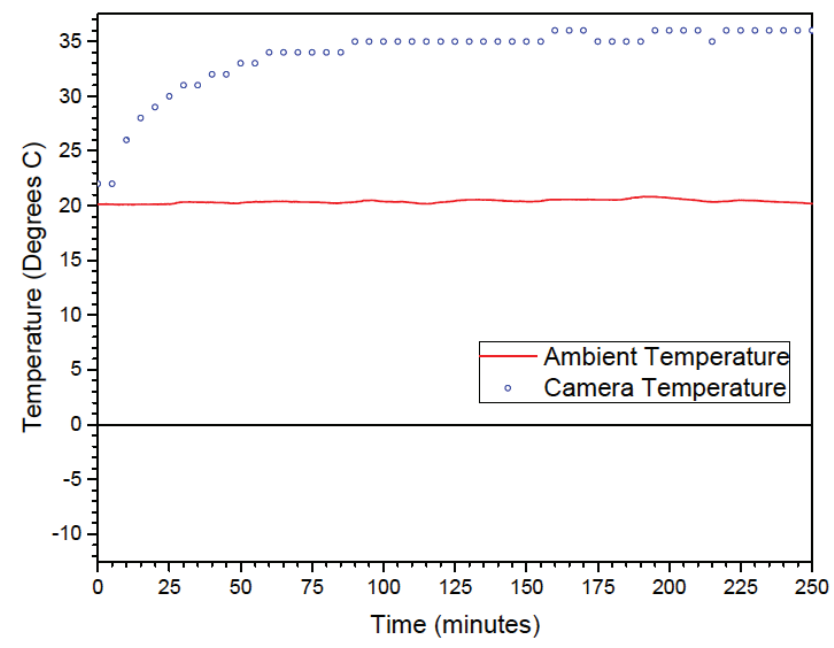

Fig. 4: The graph shows the ambient and the uncooled camera sensor during 250 minutes of five-minute test exposures, with no pause between exposures

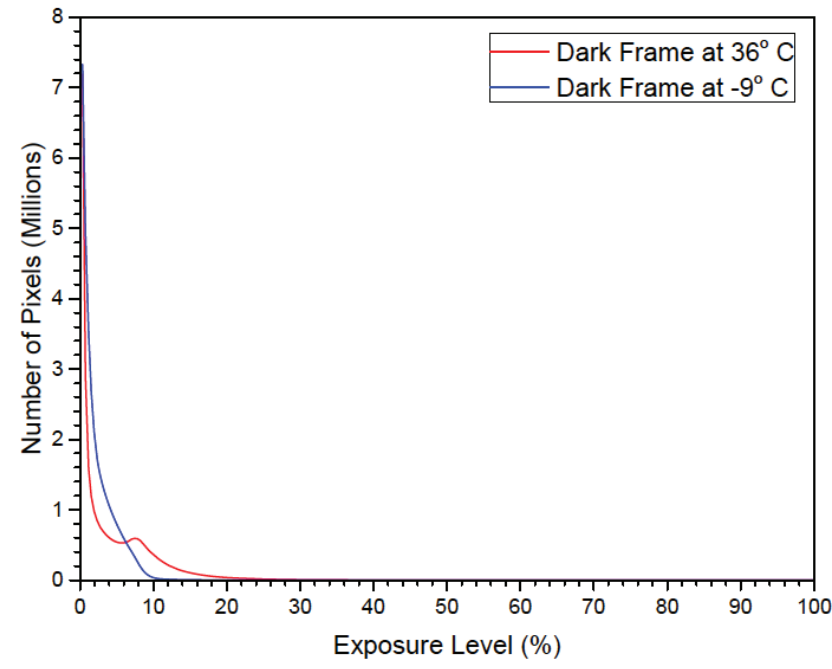

Fig. 6: This graph compares the histograms of two 15.1 megapixel 5-minute Dark Frames. One taken at $36{ }^{\circ} \mathrm{C}$ to another at $-9{ }^{\circ} \mathrm{C}$

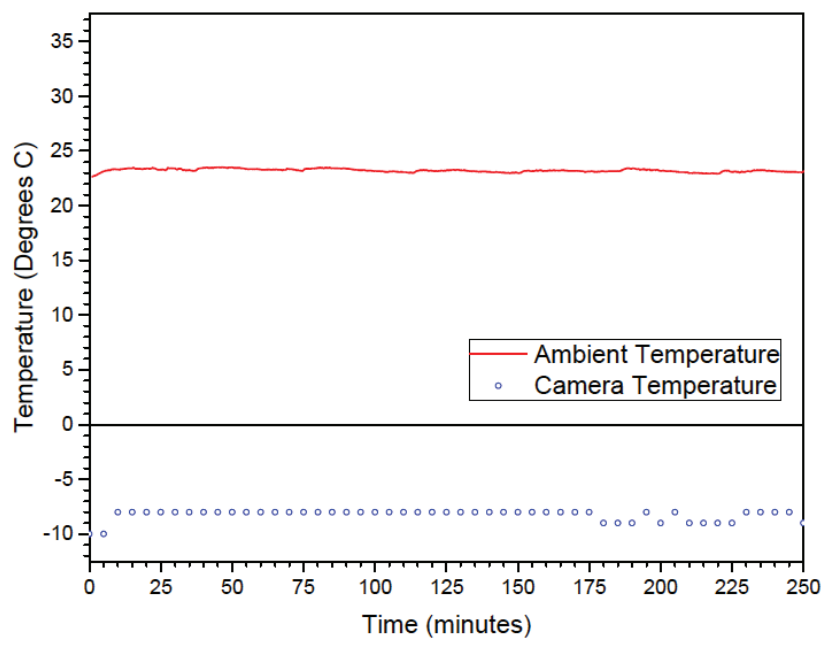

Fig. 5: The graph shows the ambient and the cooled camera sensor temperature during 250 minutes of fiveminute exposures, with no pause between exposures

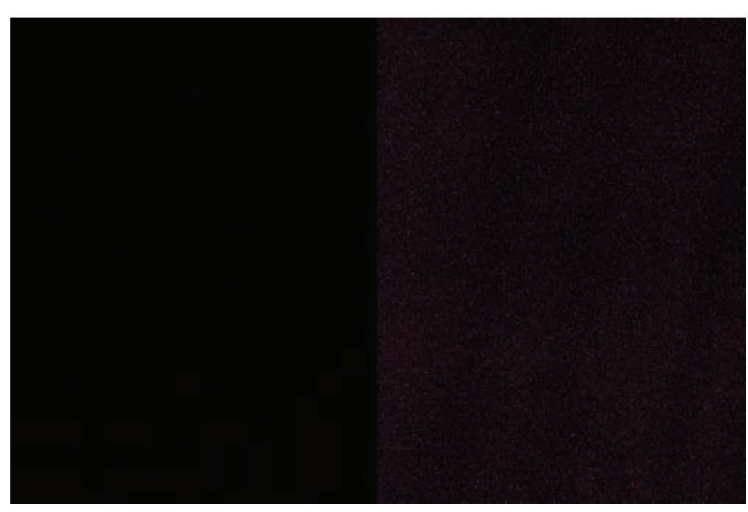

Fig. 7: The dark frames show the amount of heat noise present in two ten-minute exposures. The image on the left was taken with the cooler modification with a sensor temperature of $-9^{\circ} \mathrm{C}$ and the right image was uncooled with a sensor temp of $36^{\circ} \mathrm{C}$. 
Digital image sensors work by collecting the charge from incident photons to determine the brightness of the object being photographed. The sensors are divided into many smaller sections called picture elements (pixels) which for consumer cameras the number of pixels in a digital image sensor is measured in millions of pixels (megapixels). The histograms in (Fig. 6) show the number of pixels, 15.1 megapixels for the camera used for this project, in two Dark Frames which have registered a charge at exposure levels from zero to one hundred percent. The exposure level is the percentage of the full charge capacity for a given pixel. For a Dark Frame where light is kept from hitting the sensor, any charge collected by a pixel is the result of either heat/electrical noise or artifacts in the sensor. The heat/electrical noise in the sensor is the result of random false detections of photons from the electricity being used to operate the Fig.8: This graph compares the histograms of a Dark Frame to that of sensor and the heat it generates.

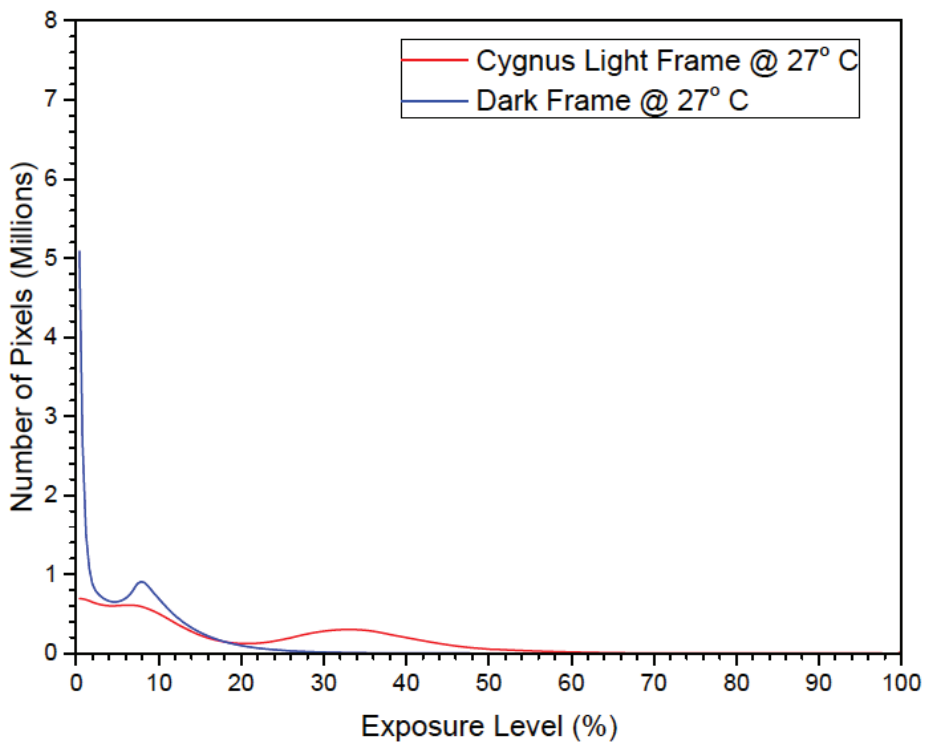

(Fig. 7) shows a comparison of samples of the images used to generate the histograms in (Fig. 6). For long exposures of faint objects such as nebulae, reducing the amount of noise present in the images helps to increase the contrast in images taken and improve the quality of the resulting images. The ability to maintain low temperatures with the cooled image sensor resulted in a significant decrease in the amount of noise in the images, shown by the histograms in (Fig. 6) along with samples of the images in (Fig. 7). An example of why reducing the noise in long exposures for studies such as the Observing Nebulosities project is shown in (Fig, 8), histograms of both a Light Frame of the Cygnus Superbubble and a corresponding Dark

Frame at the same temperature. The noise in the Dark Frame shown by the blue curve is a significant portion of the charge collected by the sensor for the Light Frame and some of the less bright portions of the Cygnus Superbubble covered in the image are lost to sensor noise.

\section{Future Work}

Now that the camera has been modified and tested, future plans include: collecting field data and improving the cooler design. More data will be collected on the Cygnus Superbubble to compare image quality before and after the camera modification. The current plan to improve the cooler design is to remove the heatsink between the TEC and the back of the image sensor and place a thin thermistor between them. This should remove some of the temperature gradient between the image sensor and TEC and allow for more accurate monitoring of the camera temperature by the Arduino. 
References

Burton, M. 2016. “Arduino Temperature Control Library” [Source Code].

https://github.com/ milesburton/Arduino-Temperature-Control-Library.

Caplan, J., Deharveng, L., 1985. “Absolute H-alpha and H-beta photometry of LMC HII regions.” Astronomy \& Astrophysics Supplement Series, 62, 63. http://adsabs.harvard.edu/abs/1985A\&AS...62...63C

Christopherson, C., Kaltcheva, N., 2015. “Observing Nebulosities: The Cygnus Superbubble.” Proceedings of the 25th Annua 1 Wisconsin Space Conference.”Cox, P., Deharveng, L., Caplan, J. 1987. "Extinction and reddening towards compact Galactic HII regions.” Astronomy \& Astrophysics, 171, 277. http://adsabs.harvard.edu/abs/1987A\&A...171..277C

Fried, L., 2016. “Adafruit HDC1000 Library” [Source Code]. https://github.com/adafruit/ Adafruit_HDC1000_Library.

Gruendl, R. A., Chu, Y. H., Dunne, B. C., \& Points, S. D. 2000. “A morphological diagnostic for dynamical evolution of Wolf-Rayet bubbles.” The Astronomical Journal, 120(5), 2670. http://dx.doi.org/10.1086/316830

Starizona. 2014. “Calibration Images: Advanced Imaging Techniques.” http://starizona.com/acb/ ccd/advimcal.aspx.

Studt, J., et al. 2014. "OneWire Library” (Version 2.2) [Source code]. https://github.com/ PaulStoffregen/OneWire. 